\title{
Temperature and cholera toxin B are factors that influence formation of membrane nanotubes in RT4 and T24 urothelial cancer cell lines
}

This article was published in the following Dove Press journal:

International Journal of Nanomedicine

II March 201]

Number of times this article has been viewed

\author{
Doron Kabaso ${ }^{*}$ \\ Maruša Lokar ${ }^{*}$ \\ Veronika Kralj-Iglič ${ }^{2}$ \\ Peter Veranič ${ }^{3}$ \\ Aleš Iglič
}

'Laboratory of Biophysics, Faculty of Electrical Engineering, 'Laboratory of Clinical Biophysics, Faculty of

Medicine, ${ }^{3}$ Institute of Cell Biology Faculty of Medicine, University of Ljubljana, Ljubljana, Slovenia; *These two authors equally share the first authorship
Correspondence: Doron Kabaso Laboratory of Biophysics, Faculty of Electrical Engineering, University of Ljubljana, Ljubljana, Slovenia Tel +386 | 4768825

Fax +386 I 4768850

Email doron.kabaso@fe.uni-lj.si

\begin{abstract}
The growth of membrane nanotubes is crucial for intercellular communication in both normal development and pathological conditions. Therefore, identifying factors that influence their stability and formation are important for both basic research and in development of potential treatments of pathological states. Here we investigate the effect of cholera toxin B (CTB) and temperature on two pathological model systems: urothelial cell line RT4, as a model system of a benign tumor, and urothelial cell line T24, as a model system of a metastatic tumor. In particular, the number of intercellular membrane nanotubes (ICNs; ie, membrane nanotubes that bridge neighboring cells) was counted. In comparison with RT4 cells, we reveal a significantly higher number in the density of ICNs in T24 cells not derived from RT4 without treatments $(P=0.005)$, after 20 minutes at room temperature $(P=0.0007)$, and following CTB treatment $(P=0.000025)$. The binding of CTB to GM1-lipid complexes in membrane exvaginations or tips of membrane nanotubes may reduce the positive spontaneous (intrinsic) curvature of GM1-lipid complexes, which may lead to lipid mediated attractive interactions between CTB-GM1-lipid complexes, their aggregation and consequent formation of enlarged spherical tips of nanotubes. The binding of CTB to GM1 molecules in the outer membrane leaflet of membrane exvaginations and tips of membrane nanotubes may also increase the area difference between the two leaflets and in this way facilitate the growth of membrane nanotubes.
\end{abstract}

Keywords: cancer cells, membrane nanotubes, cholera toxin

\section{Introduction}

Membrane nanotubes, as a novel intercellular communication mechanism, are extensively studied both structurally and functionally. They were found naturally occurring in many cell types (reviewed in ${ }^{1-5}$ ), and categorized into several types by different authors with respect to their proposed function and structural characteristics. ${ }^{6-8}$ The number of membrane nanotubes per cell may be affected by the cell membrane elasticity and composition, in which interacting anisotropic membrane constituents (proteins, lipids, or their complexes) could bend the cell membrane and stabilize highly curved anisotropic membrane regions. ${ }^{9-12}$ In particular, the clustering of anisotropic membrane constituents into lipid rafts at the outer leaflet of a cell membrane might lead to the outward bending of a membrane and to the initial growth of a membrane protrusion. ${ }^{13}$

Previous X-ray crystallography studies have revealed that cholera toxin from Vibrio cholerae consist of a toxic subunit A and a nontoxic pentameric subunit $\mathrm{B}$. Subunit B binds to its receptor, pentasaccharide GM1, binding one GM1 molecule per subunit B monomer, while subunit A resides upon subunit B. ${ }^{14}$ After binding to GM1 molecules, which are localized in lipid rafts at the outer leaflet of the cell 
membrane, these complexes are internalized and transported to the endoplasmatic reticulum and Golgi apparatus. In a cholera toxin, the A subunit is cleaved at the Golgi to yield the active derivative of the cholera toxin. ${ }^{14-16}$ In the present study, the effects of adding the nontoxic cholera toxin B (CTB) (without the A subunit) extracellularly on the stability and growth of membrane nanotubes was being investigated in nonmalignant and malignant urothelial cancer cell lines.

We report the differences in cell shape and intercellular membrane nanotube (ICN) numbers between a nonmalignant urothelial cell line RT4 and a malignant urothelial cell line T24. The RT4 cell line derives from a transitional cell papilloma of urinary bladder, in which cells are not malignant, demonstrating growth and motility characteristics of normal epithelial cells. In contrast, T24 cells are transitional cell carcinoma of urinary bladder, wherein cells show malignant behavior, spreading and separating during cell motility. The progression from a benign cell line to a malignant cell line has been correlated with specific mutations. ${ }^{17}$ Therefore, the comparison between the benign and malignant model systems reveals a continuous process, in which the observed higher number of ICNs in T24 cell lines can be considered as an increase during the development of a urothelial cancer.

CTB is a pentameric subunit of cholera toxin of Vibrio cholerae that binds specifically to the branched pentasaccharide moiety of lipid ordered domain resident entity (ganglioside GM1) on the surface of target human intestinal epithelial cells. ${ }^{14-16}$ Therefore, CTB in conjunction with antibodies to CTB (anti-CTB) is widely used as a marker for lipid ordered domains in cells. We suggest that the addition of CTB causes the lateral clustering of GM1 lipid rafts in the outer leaflet of a cell membrane. As a result, the membrane spontaneous curvature is locally changed, facilitating the formation of membrane protrusions in these membrane regions. The addition of anti-CTB reduces the number of membrane nanotubes, which might be due to the formation of CTB-GM1-anti-CTB protein network of nonzero shear elasticity that cannot be deformed into long tubes. ${ }^{18}$

Since cell metabolism determines at least partially the growth of membrane nanotubes, a change in the temperature of the growth medium may affect the expression level of membrane nanotubes. A previous experimental study showed that temperature treatment could cause phase separation of GM1 molecules, changing the diameter of giant unilamellar vesicles (GUVs). ${ }^{19}$ Therefore, it is crucial to elucidate the effects of temperature on ganglioside (eg, GM1) distributions and on the formation of membrane nanotubes.

\section{Materials and Methods Cell culture}

Urothelial cell lines RT4 (Figure 1A) and T24 (Figure 1B) were cultured in a 1:1 mixture of advanced Dulbecco's modified Eagle's medium (Gibco, Invitrogen, Carlsbad, CA) and Ham's F 12 (Sigma-Aldrich, St. Louis, MO), supplemented with $10 \%$ fetal calf serum (Gibco, Invitrogen), $5 \mu \mathrm{g} / \mathrm{mL}$ insulin, $5 \mu \mathrm{g} / \mathrm{mL}$ transferrin, $100 \mathrm{mg} / \mathrm{mL}$ hydrocortisone and $5 \mathrm{ng} / \mathrm{mL}$ selenite (Gibco, Invitrogen), $1800 \mathrm{U} / \mathrm{mL}$ cristacyclin (Pliva, Zagreb, Croatia), and $0.222 \mathrm{mg} / \mathrm{mL}$ streptomycinesulfate (Fatol Arzneilmittel GmbH, Schiffweiler, Germany). Cells were incubated at $37^{\circ} \mathrm{C}$ in a humidified incubator in an atmosphere of $5 \% \mathrm{CO}_{2}$.

Prior to the experiments cells were seeded onto sterile glass coverslips (Brand GmbH, Wertheim, Germany) and allowed to grow to approximately $40 \%$ to $50 \%$ and $70 \%$

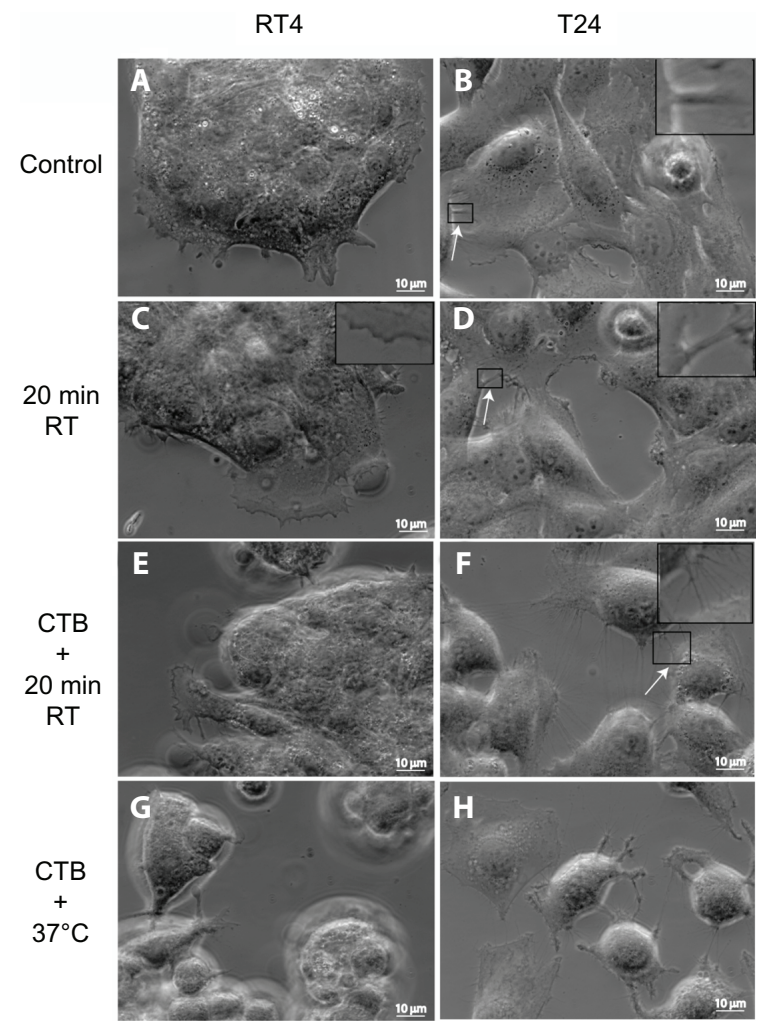

Figure I Phase-contrast microscope images of RT4 and T24 cells treated with either cholera toxin B (CTB) or temperature. Images of RT4 A) and T24 B) cells growing in normal conditions without any treatments (control experiments). Note that intercellular membrane nanotubes (ICNs) are not present between RT4 cells, while a few are present between T24 cells (arrow). Following their growth in normal conditions, the cells are moved to room temperature (RT) for 20 minutes, showing no significant change in the morphology of RT4 cells C), while T24 cells become more separated D). In conjunction with 20 minutes at RT, cells are treated with CTB. Note that in RT4 cells E) no ICNs are observed, while many ICNs (arrow) are present between T24 cells F). Finally, the combination of CTB and temperature incubation at $37^{\circ} \mathrm{C}$ induces rounding of RT4 cells and their detachment $\mathbf{G}$ ), while T24 cells demonstrate large numbers of ICNs $\mathbf{H}$ ). 
to $80 \%$ confluency. T24 cell line was grown overnight and RT4 cells were left to grow for 48 to 72 hours.

\section{Temperature dependence and CTB experiments}

For controls, the growth medium was removed, and the cells were briefly washed with phosphate-buffered saline (PBS) warmed to $37^{\circ} \mathrm{C}$ and immediately fixed with $2 \%$ formaldehyde in PBS warmed to $37^{\circ} \mathrm{C}$. A second set of cells was left in a humidified chamber at room temperature, approx. $23^{\circ} \mathrm{C}$, for 20 minutes in normal growth medium. Afterwards medium was removed and cells were washed and fixed in $2 \%$ formaldehyde in PBS at room temperature for 30 minutes.

A stock solution of fluorescent CTB conjugate (component A of Vybrant Alexa Fluor 488 Lipid Raft Labeling Kit [Molecular Probes, Invitrogen, Carlsbad, CA]) was diluted to a final concentration of $4 \mu \mathrm{g} / \mathrm{mL}$ in PBS and added to the washed cells on glass coverslip. Cells were incubated in CTB solution for 20 minutes either at room temperature or at $37^{\circ} \mathrm{C}$ in humidified chamber, then washed with PBS and fixed in $2 \%$ formaldehyde in PBS for 30 minutes.

We followed the manufacturer's protocol for labeling cells with anti-CTB antibodies. The temperature of PBS was always adapted to the temperature conditions of the experiments.

\section{Actin labeling, immunofluorescence labeling, and microscopy}

Cells treated with CTB were washed with PBS and fixed in $2 \%$ formaldehyde (Merk Schuthand, OHG, Germany) for $30 \mathrm{~min}$ utes (15 minutes for caspase-3 labeling). After washing with PBS, the cover slips were incubated in $0.25 \%$ Triton X-100 in PBS for 6 minutes at room temperature, washed 3 times in PBS, and incubated in $0.33 \mathrm{M}$ sucrose in $0.2 \mathrm{M}$ cacodylate buffer for 30 minutes. Samples were blocked in 2\% bovine serum albumin (BSA; Sigma-Aldrich) in $0.2 \% \mathrm{NaN}_{3}$ in PBS for 30 minutes at RT. Coverslips with cells were incubated in primary antibodies for 1 hour at $37^{\circ} \mathrm{C}$ or overnight at $4{ }^{\circ} \mathrm{C}$, then washed in PBS for 10 minutes and incubated in secondary antibodies for 30 minutes at $37^{\circ} \mathrm{C}$. Actin labeling in $16.7 \mu \mathrm{g} / \mathrm{mL}$ phalloidin (phalloidin-FITC) (Sigma-Aldrich) in $20 \%$ methanol (Carlo Erba, Italy) in PBS for 30 minutes was performed after secondary antibody incubation and $10 \mathrm{~min}-$ utes washing in PBS. Afterwards coverslips were decanted and embedded in vectashield-4',6-diamidino-2-phenylindole (vectashield-DAPI) (Vector Laboratories, Peterborough, UK), and analyzed in a fluorescence microscope Axio-Imager
Z1 microscope (Carl Zeiss Inc., USA). For active caspase-3 labeling rabbit polyclonal antibodies (AbCam Ltd, UK) were used at concentration $15 \mu \mathrm{g} / \mathrm{mL}$. As secondary antibodies goat anti-mouse antibodies conjugated with tetramethylrhodamine isothiocyanate (TRITC) and the goat anti-rabbit antibodies conjugated with Alexa-Fluor ${ }^{\circledR} 555$ (Molecular Probes, Invitrogen) were used.

\section{Phase-contrast and fluorescence image acquisition}

Cells were analyzed in a fluorescence microscope Axio-Imager Z1 microscope (Carl Zeiss Inc., New York, NY). Phase-contrast images were taken with $63 \times$ water immersion objective (numerical aperture $[\mathrm{NA}]=0.95$ ) and fluorescence images with Plan-Apochromat $63 \times(\mathrm{NA}=1.4$, oil $)$.

\section{Morphometric analysis}

Sampling was performed as follows: a cover glass with fixed cells was imaged with water immersion objective $(63 \times)$. Images were taken at every second visual field, second horizontal, and second vertical axis. Afterwards 30 randomly chosen images were analyzed for each treatment by counting the cells and ICNs per image. Only membrane nanotubes that made contact between neighboring cells were counted as ICNs. The ICN density, ie, the number of ICNs per cell, was calculated as: ICN density $=$ (total number of ICNs/total number of cells).

\section{Testing for apoptosis}

To assess whether exposing the cells to temperature treatment or the addition of CTB to RT4 and T24 cells induced apoptosis, cells were immunostained for active caspase- 3 as a marker of apoptosis. The integrity of actin filaments was evaluated by labeling with phalloidin-fluorescein isothiocyanate (FITC) and the nucleus was labeled with DAPI.

\section{Statistical analysis}

All statistical comparisons between RT4 and T24 groups were performed using a $t$-test for independent samples.

\section{Results \\ Control experiment}

We compared two urothelial cancer cell lines: 1) the first was a nonmalignant cell line, RT4, which had a similar phenotype to normal cells; and 2) the second was a malignant cell line, T24, where cells were spread apart and showed extensive motility. The RT4 cell lines were grown in normal growth medium, 
and fixed with prewarmed formaldehyde (Figure 1A). Note that the cell islands were tightly connected to each other, and that the cells at the edge of a cell island formed short filopodia. In addition, there were few ICNs present between cells, mostly at the edges of the cell island.

The T24 cell lines were also grown in normal growth medium at $37^{\circ} \mathrm{C}$ and fixed with prewarmed formaldehyde (Figure 1B). In contrast to RT4 cells, T24 cells grew partly one on top of the other (Figure 1B). Cells at the middle of the cell island were tightly connected by ICNs. The number of ICNs in T24 cells highly exceeded the number of ICNs in RT4 cells (Figure 1B, Figure 2).

\section{Incubation at room temperature for 20 minutes}

Following the growth of RT4 cells in normal conditions, the change in temperature to room temperature for 20 minutes did not cause any significant change in their morphology (Figure 1C), while the cell edge retracted, forming a wave-like shape. In contrast, the same treatment of T24 cell lines caused a clear rounding of cells, occasionally forming ICNs between cells (Figure 1D).

\section{CTB + incubation at room temperature for 20 minutes}

The shape of RT4 cells did not significantly change after treatment of CTB for 20 minutes at room temperature (Figure 1E). The CTB-induced effects included rounding of the cell edge, formation of irregular protrusions, and formation of few ICNs. On the other hand, T24 cells became more rounded, and irregular protrusions and ICNs were formed

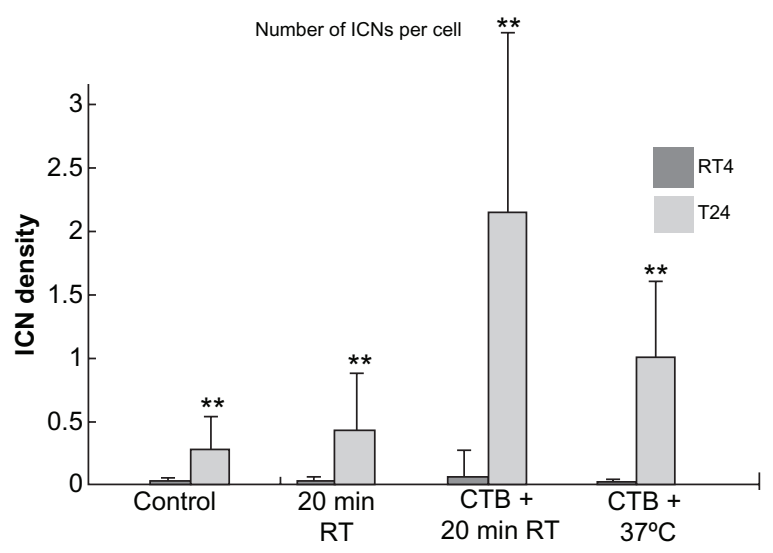

Figure 2 Summary statistics of changes in intercellular membrane nanotube (ICN) densities of treated RT4 and T24 cell lines. The control case was not treated. Note that both 20 minutes at room temperature (RT) and cholera toxin B (CTB) treatments significantly $(P<0.0$ I**) increase the ICN density among T24 cells compared with among RT4 cells.

Note: Data are means \pm standard deviation. along the edges as a result of CTB treatment (Figure 1F). The T24 cells were rather heterogeneous in binding CTB and their responses to it, while no such heterogeneity was observed in RT4 cells.

\section{$\mathrm{CTB}+$ incubation at $37^{\circ} \mathrm{C}$}

In RT4 cells, CTB induced rounding of the cells and their detachment from the substratum (Figure 1G). The cell detachment and acquisition of spherical shape was expressed even more in T24 cells after CTB treatment at $37^{\circ} \mathrm{C}$. In addition, irregular protrusions poked upward from the cell surface, while numerous ICNs connected 2 or more adjacent cells (Figure 1H).

\section{Morphometric analysis}

To evaluate the effect of CTB and temperature treatments in RT4 and T24 cells, the numbers of cells and of ICNs were counted in acquired images. The ICN density was calculated in each image. Summary statistics for the differences in ICN density between RT4 and T24 cells after different treatments are shown in Figure 2 and Table 1. Without any treatment there was a significant increase in ICN density of T24 cells compared with RT4 cells (RT4 $=0.0165 \pm 0.0415 ;$ T24 $=0.2667 \pm 0.3917, P<0.01$ ). Due to additional 20 minutes at room temperature, the ICN density was significantly greater in T24 cells $(\mathrm{RT} 4=0.0114 \pm 0.0476 ; \mathrm{T} 24=0.4157 \pm 0.5483, P<0.01)$, indicating the susceptibility of T24 cells to change in temperature. CTB treatment in conjunction with 20 minutes of incubation at room temperature significantly increased ICN density (RT4 $=0.0458 \pm 0.2668 ;$ T2 $4=2.1006 \pm 2.6136$, $P<0.01)$. In addition, the combined treatment of CTB and physiologic temperature $\left(37^{\circ} \mathrm{C}\right)$ showed a deleterious effect on ICN numbers and cell count (RT4 $=0.0012 \pm 0.0052$; $\mathrm{T} 24=0.9656 \pm 0.6665, P<0.01)$, which was due to cell damage and not apoptosis (Figure 3 ).

Table I Statistical data on the density of ICNs for the different treatments in RT4 and T24 cells

\begin{tabular}{lllll}
\hline Cell type & Treatment & Number & Mean & SD \\
\hline RT4 & Control & 21 & 0.0165 & 0.0415 \\
RT4 & 20 minutes RT & 24 & 0.0114 & 0.0476 \\
RT4 & CTB +20 minutes RT & 34 & 0.0458 & 0.2668 \\
RT4 & CTB +20 minutes $37^{\circ} \mathrm{C}$ & 20 & 0.0012 & 0.0052 \\
T24 & Control & 34 & 0.2667 & 0.3917 \\
T24 & 20 minutes RT & 33 & 0.4157 & 0.5483 \\
T24 & CTB +20 minutes RT & 28 & 2.1006 & 2.6136 \\
T24 & CTB +20 minutes $37^{\circ} \mathrm{C}$ & 30 & 0.9656 & 0.6665 \\
\hline
\end{tabular}

Abbreviations: $\mathrm{RT}$, room temperature; ICNs, intercellular membrane nanotubes; CTB, cholera toxin $B$. 


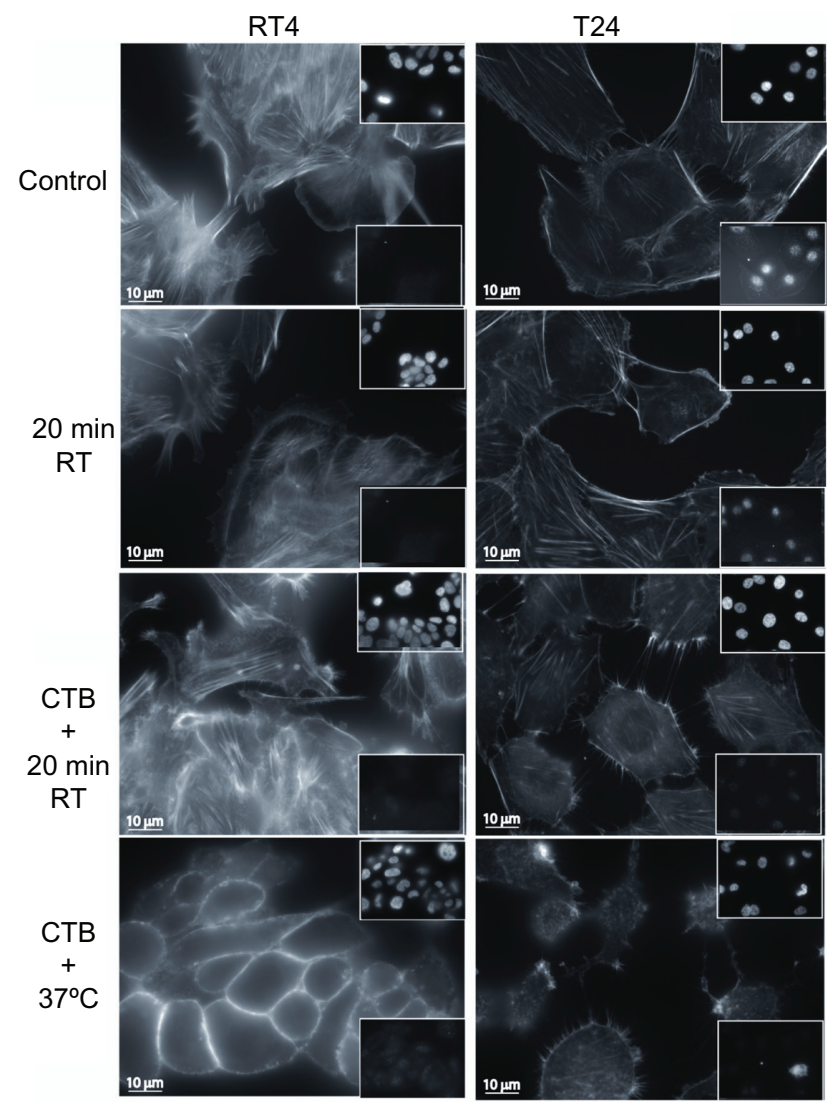

Figure 3 Cell testing for apoptosis using actin filament and nucleus fluorescence markers. To assess whether exposing cells to an ambient temperature or addition of cholera toxin B (CTB) to RT4 and T24 cells induced apoptosis, cells are immunostained for the integrity labeling of actin filaments with phalloidin-fluorescein isothiocyanate, the integrity of the nucleus with vectashield-4',6-diamidino-2phenylindole (top insets), and the integrity of the cells using caspase- 3 (bottom insets). Note that neither temperature nor CTB treatments trigger apoptosis in RT4 and T24 cells. However, CTB does cause cell damage in some cells, mostly at $37^{\circ} \mathrm{C}$.

\section{Testing for apoptosis}

To assess whether exposing RT4 and T24 cells to temperature treatment or whether the addition of CTB induced apoptosis, cells were immunostained for active caspase-3. As a marker for cell shape and integrity labeling of actin filaments, phalloidinFITC was added, and as a marker for the nucleus, DAPI was used (Figure 3). Lowering the temperature to room temperature did not trigger apoptosis at the given experimental conditions in RT4 or T24 cells, whereas CTB did cause cell damage in some $\mathrm{T} 24$ cells treated with $\mathrm{CTB}$ at $37^{\circ} \mathrm{C}$ (Figure 3 ).

\section{Reversing the induced growth of membrane nanotubes}

Binding of CTB to the cell membrane of RT4 (Figure 4A) and T24 (Figure 4B) induced growth of thin specific membrane nanotubes with semivesicular structures at the ends of the tips attached to the substratum (arrows). Moreover, some of the induced membrane nanotubes made contact with

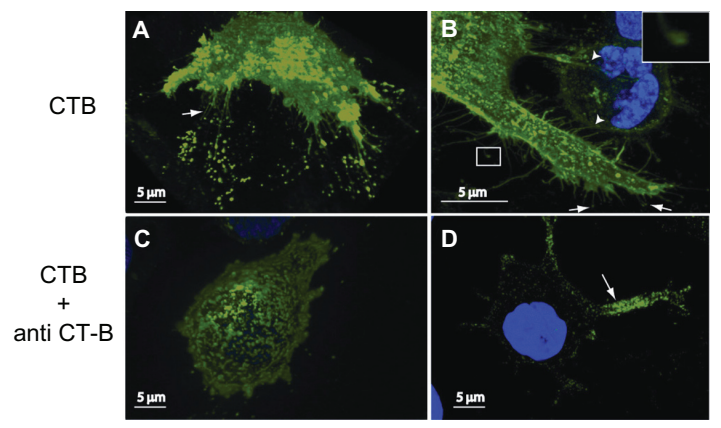

Figure 4 Reversing the induced growth of membrane nanotubes by the addition of anti-cholera toxin B (CTB). The addition of CTB to RT4 A) and T24 B) cells reveals the growth of membrane nanotubes (arrows), as well as membrane lipid rafts (marked by fluorescent CTB-GMI complexes) at the tips and entire length of membrane nanotubes. Note that some membrane nanotubes (ICNs) make contact with a neighboring T24 cell (b; arrowheads), and the presence of a microvesicular structure at the tip of membrane nanotubes (close up view). The addition of anti-CTB causes the retraction of membrane nanotubes in both RT4 C) and T24 D) cells.

a neighboring cell (Figure 4B; arrowheads). Staining of the cell membrane was more or less uniform. Subsequently, binding of anti-CTB almost entirely abolished tubulation of the membrane, leading to patchy staining of the membrane (Figures 4B, D). Anti-CTB (pentameric antibodies) might cross-link (intercalate) CTB units, facilitating phase separation of lipids and proteins into more stiff microdomains with nonzero shear elastic modulus, which do not allow the growth of membrane nanotubes in RT4 (4B), and T24 cells (4D).

\section{Possible mechanisms for CTB-facilitated membrane nanotube formation}

To explain the induced growth of membrane nanotubes with semivesicular structures at their tip following the addition of CTB, we propose that the binding of CTB to GM1-lipid membrane complexes at the outer leaflet of the cell membrane increases the local area difference between the two leaflets. The formation of GM1-lipid complexes at the tip of membrane nanotubes may affect the membrane curvature. The initial aggregation of GM1 molecules may contribute to the formation of a membrane protrusion of positive curvature (Figure 5B). The accumulation of a few
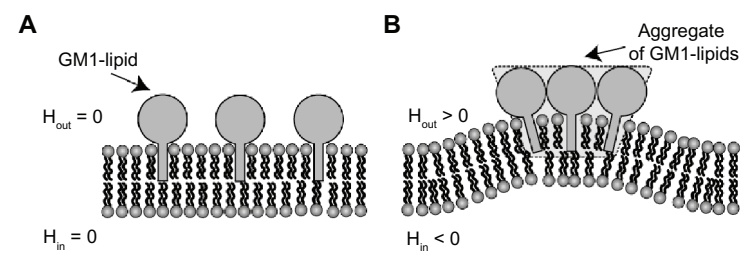

Figure $\mathbf{5}$ Schematic diagram for the effects of GMI interaction on membrane curvature. Single GMI molecules have zero intrinsic (spontaneous) curvature A). Small membrane protrusions are formed due to the interaction between GMI molecules, where it is assumed that small GMI aggregates have positive intrinsic curvature $\mathbf{B}){ }^{27,28} \mathrm{H}_{\text {out }}$ and $\mathrm{H}_{\text {in }}$ are the mean curvature of the outer and inner cell membrane leaflets, respectively. 
aggregates may further bend the membrane, contributing an additional protrusive force. On the other hand, the addition of flat CTB and the formation of less curved CTB-GM1 nanodomains would reduce the spontaneous curvature of the tip region of the nanotubes, explaining the observed vesicular region at the tips of membrane nanotubes (Figure 7C).

\section{The model}

To test the hypothesis that the initial formation of a membrane nanotube is driven by the aggregation of CTB-GM1 complexes (Figure 7B), we constructed a model to study the effects of strong binding of CTB to GM1 molecules. The purpose of our model is to test whether the positive spontaneous curvature of CTB-GM1 complexes and the strong binding of GM1s to CTB are sufficient to drive a membrane instability causing the growth and coalescence of membrane protrusions (Figure 7).

This model was based on our previous model, in which the membrane curvature was coupled to spontaneous curvature of membrane inclusions. ${ }^{20-22}$ According to the radius of curvature at the tip of membrane nanotubes, the spontaneous curvature of CTB-GM1 complexes was approximated by a positive value of $5 \mu^{-1}$. The underlying assumption of the present model was that membrane inclusions, ie, CTB-GM1 complexes, contributed negative binding (interaction) energy $(-\alpha n)$. For the sake of simplicity, the modeled membrane contour of RT4 or T24 cells was considered to be nearly flat. The free energy of the model was:

$$
\begin{aligned}
F= & \int\left(\frac{1}{2} \kappa(H-\bar{H} n)^{2}+(\sigma-\alpha n)+\gamma h^{2}\right. \\
& \left.+k_{B} T n_{s} n \ln (n)-J n^{2}+\frac{J}{n_{s}}(\nabla n)^{2}\right) d s,
\end{aligned}
$$

where $\kappa$ is the membrane bending rigidity, $n$ is the density of CTB-GM1 complexes, $H$ is the local mean membrane curvature, $\bar{H}$ is the parameter describing the intrinsic mean curvature of the CTB-GM1 complexes, $n$ is the area fraction of CTB-GM1 complexes, $n \bar{H}$ is the spontaneous curvature of CTB-GM1 complexes, $n_{s}$ is the saturation density, $\sigma$ is the membrane surface tension, $\alpha$ is a proportionality constant describing the binding energy between CTB-GM1 complexes, $\gamma$ is a restoring spring due to the force of the cytoskeleton, $J$ is the direct nearest-neighbor interaction energy between CTBGM1 complexes, and $d s=d_{m} \cdot d l$ is an element of membrane area, where $d_{m}$ is the dimension of membrane perpendicular to the contour and $d l$ is a line element along the contour. $h(x)$ is the magnitude of small deformations from the flat membrane.
The first term describes the bending energy due to the mismatch between the membrane curvature and the spontaneous curvature of the CTB-GM1 complex. The second term describes the effective membrane surface tension and the binding energy between CTB-GM1 complexes. The third term describes an external trapping of the membrane, which can be represented as the force of the cytoskeleton inside the cell. The fourth term gives the entropic contribution due to the lateral thermal motion of CTB-GM1 complexes in the membrane in the limit of small $n$. The fifth term describes the nearest neighbor attractive interactions between CTB and GM1, and the sixth term prevents the sharp changes in the CTB-GM1 density. The derivation leading to Eq. (1) is a one-dimensional version of the more general expressions derived in previous studies. ${ }^{23,24}$ These expressions recover the familiar form for small undulations of a flat membrane in the Monge gauge form. See the supplementary material for the membrane contour forces and fluxes derived from the derivation of the free energy as well as the list of parameter values incorporated in our model.

Results of our numerical simulations revealed that the coupling between the CTB-GM1 density and the membrane curvature led to the formation of membrane protrusions, which coalesced into a larger membrane protrusion (Figure 6). Figure 6 shows how the initial straight membrane contour evolves from a multiprotrusion shape (dotted line) at intermediate time to a coalesced membrane protrusion (solid line) at steady state (Figure 6B). Furthermore, the

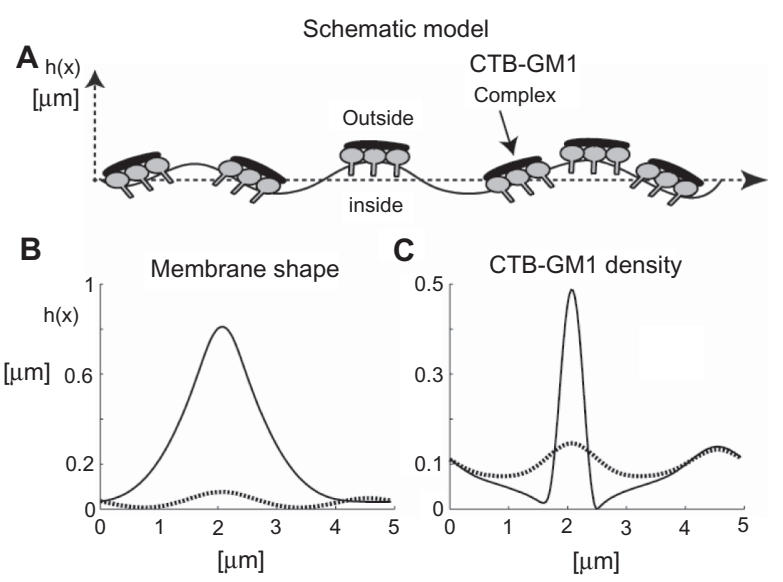

Figure 6 The effects of cholera toxin B (CTB) binding to GMI molecules on membrane shape and filament densities. A) The schematic model is a nearly straight membrane contour, which describes a segment of an RT4 or T24 cell. Numerical simulations of the flat shape membrane reveal the steady state shapes of membrane amplitudes B) and CTB-GMI densities C), given that $\alpha=0.013$ and $\bar{H}=5 \mu \mathrm{m}^{-1}$ (see Supplementary material for more information). The amplitude $h(x)$ is the value of the membrane deformation. The initial condition is a flat membrane with a random perturbation of small amplitudes $(<1 \%)$ in the CTB-GMI density around the uniform value of $n_{0}=0.1$. The intermediate time (in dotted line) is after 400 seconds, and the steady state time (in bold line) is reached within 600 seconds. 
CTB-GM1 density distribution demonstrates a similar evolution to the one observed in the membrane shape dynamics (Figure 6C).

\section{Discussion}

Numerous studies have revealed the growth of membrane nanotubes in different cell types under normal and pathological states. ${ }^{1-8}$ In particular, previously it has been shown that ICNs that connect between neighboring cells are frequently observed in malignant (transformed) cancer cell lines (T24) of urothelial origin. ${ }^{4,8}$ So far, the comparison of ICN density between nonmalignant and malignant cancer cell lines was not investigated. We here report the differences in cell shape and ICN density between nonmalignant (RT4) and malignant (T24) urothelial cell lines not only in normal conditions, but also during the application of CTB and temperature (Figure 1). Statistical analyses reveal that the ICN density in T24 cells is significantly increased compared with RT4 cells in normal conditions $(\mathrm{RT} 4=0.0165 \pm 0.0415 ; \mathrm{T} 24=0.2667 \pm 0.3917$, $P<0.01)$. Furthermore, significant increases in ICN densities were observed during CTB treatment at physiologic temperature $(\mathrm{RT} 4=0.0012 \pm 0.0052 ; \mathrm{T} 24=0.9656 \pm 0.6665$, $P<0.01)$, and during CTB treatment at room temperature $(\mathrm{RT} 4=0.0114 \pm 0.0476 ;$ T24 $=0.4157 \pm 0.5483, P<0.01)$ (Figure 2, Table 1). In addition, T24 cells are of irregular shapes and separated, which makes them morphologically different from the benign phenotype of RT4 cells, which are firmly bound to each other (Figure 1). Testing for cell apoptosis using fluorescence markers for actin filaments and the nucleus demonstrated that neither lowering the temperature nor the addition of CTB triggered apoptosis in RT4 or T24 cells (Figure 3 ). In addition, the induced growth of membrane nanotubes can be reversed or prevented by the application of CTB antibodies (anti-CTB) (Figure 4).

Based on the experiment data, we discuss the following hypotheses. Firstly, the observed increases in ICN densities during the transformation from benign to malignant urothelial cells could be concomitant with increases in the membrane densities of GM1 and cholesterol molecules. Secondly, based on structure data of CTB-GM1 complexes and insight from the theory of membrane elasticity, ${ }^{14,15,24}$ we suggest that the binding of CTB to GM1s reduces the spontaneous curvature of GM1 aggregates, explaining the observed increased diameter of the tip region of membrane nanotubes (Figure 4A, B). Thirdly, the clustering of GM1 molecules by CTB causes the formation of isotropic nanodomains at the outer leaflet of cell membranes, facilitating the growth of spherical membrane exvaginations (Figure 7B).
In previous studies, the effects of adding CTB were investigated using GUVs. ${ }^{25,26}$ Since the radius of GUVs is between 20 and $30 \mu \mathrm{m}$, the membrane is practically flat compared with the radius of curvature at the tip of membrane nanotubes. It has been shown that the addition of CTB to GUVs induced invagination, where CTB-GM1 complexes may stabilize a negative membrane curvature..$^{25,26}$ These results are in agreement with those of the present study, in which the addition of CTB reduced the membrane curvature, forming a spherical tip in membrane nanotubes. The underlying mechanism is due to the flat crystal structure of CTB, ${ }^{14}$ whereby binding to more than 1 GM1 molecule reduces the spontaneous curvature of a GM1 aggregate (Figure 7).

Gangliosides such as GM1s are pentasaccharides of strong hydrophilic character, ${ }^{27}$ which are docked into the outer leaflet of cell membranes by a ceramide hydrophobic moiety. It has been shown that GM1 pentasaccharides are aggregated at the highly curved edges of caveolae, where the large positive curvature is stabilized by hydrogen bonds between the sugar moieties of neighboring GM1s. ${ }^{27}$ In another study, cholera toxin (CT) and Shiga toxins have been implicated in the formation of invaginations, ${ }^{25,26}$ where it has been suggested that the positive curvature at the neck region of caveolae is stabilized by the aggregation of GM1s. ${ }^{25}$ Previous theoretical analyses demonstrated that the curvature of small nanodomains is changed when gangliosides with bulky headgroups such as GM1s come together, which is driven by the increased number of hydrogen bonds between neighboring sugar moieties. ${ }^{27,28}$ Accordingly, we propose that the aggregation of GM1 molecules may generate the positive membrane curvature mediating the initial growth of membrane nanotubes (Figures 5 and 6). Consequently, alterations in the amounts of GM1s in T24 malignant cancer cell lines may account for the observed increases of ICN densities compared with RT4 benign cancer cell lines (Figures 1 and 2).

To shed light on the mechanisms by which CTB facilitates the formation of membrane nanotubes, the model employed in the present study has shown that CTB could have a role in the initial growth of membrane nanotubes (Figure 6). In particular, the binding of more than $1 \mathrm{GM} 1$ molecule by CTB brings GM1s into close contact, facilitating the coalescence of small membrane protrusions into a single larger membrane protrusion (Figure 6). The resulting negative interaction energy between neighboring GM1s complexes may counterbalance the loss of configurational entropy during the process of lateral sorting of GM1s, driving the initial dynamical instability. ${ }^{10,20,22}$ From the crystal structure of $\mathrm{CTB},{ }^{14}$ it is possible to conclude that the native CTB has 
A

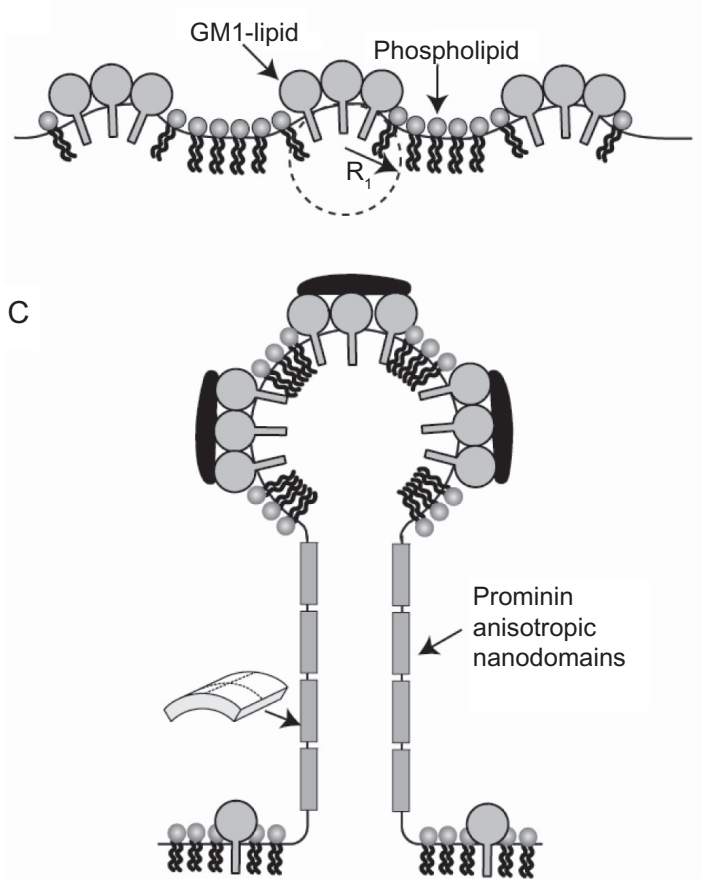

B

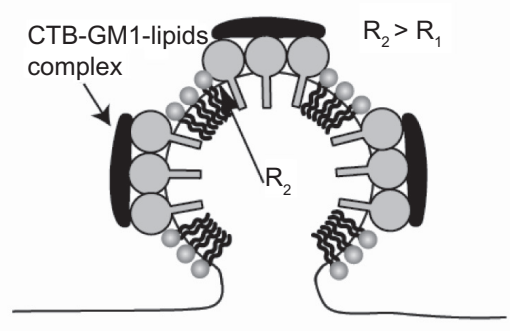

D

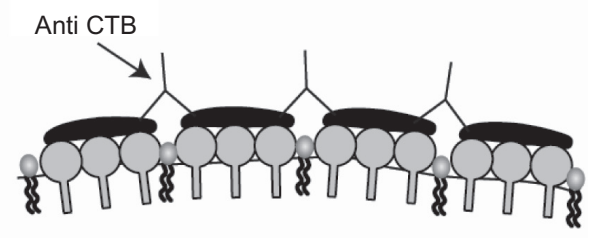

Figure 7 The effects of cholera toxin B (CTB) and prominins on the initial formation and stability of membrane nanotubes. Due to positive spontaneous curvature of GMI aggregates (see Figure 5B), such small GMI aggregates would sense and stabilize small membrane protrusions $\mathbf{A}$ ). The binding of each CTB molecule to a few GMI aggregates would lead to the aggregation of CTB-GMI complexes, thereby driving the coalescence of small membrane protrusions into a large spherical protrusion B). Due to flat shape of CTB it is assumed that bound CTB reduces the spontaneous curvature of GMI aggregates. The neck region of the membrane protrusion attracts anisotropic membrane nanodomains such as prominins, which facilitate and stabilize the growth of membrane nanotubes C). The addition of anti-CTB (pentameric antibodies) may cross-link (intercalate) CTB units, thereby leading to the formation of stiff microdomains that do not allow the growing of membrane nanotubes $\mathbf{D}$ ).

a flat shape. Consequently, the spontaneous curvature of CTB-GM1 complexes is less positive than the spontaneous curvature of pure GM1 complexes (Figure 5). The present dynamic model shows that a positive spontaneous curvature of CTB-GM1 could drive a positive feedback loop in which the aggregation of CTB-GM1 complexes would not only bend the membrane but also lead to further attraction of CTB-GM1 complexes. This relationship is evident from the mirror image of CTB-GM1 densities and membrane amplitude distributions (Figure 6B, C).

The growth of long membrane protrusions is not favorable for the Helfrich bending energy along anisotropic nanotubular regions. It has been demonstrated that the mild depletion of cholesterol and the degradation of actin filaments do not impair the stability of nanotubular structures. ${ }^{8,10}$ This stability could be due to prominin anisotropic nandomains along the tubular regions of membrane nanotubes (Figure 7C). Prominins may also reduce the anisotropic membrane bending energy, facilitating the growth of membrane nanotubes. ${ }^{5,8,10}$

Besides the curvature-generation mechanism of GM1s initiating the formation of membrane nanotubes, GM1s may also have an indirect effect through the recruitment of I-BAR domain proteins attached to the inner membrane surface (Figure 8). Previous studies have shown that I-BAR domain proteins, bound to the inner leaflet, can generate a negative membrane curvature and mediate the actin nucleation machinery. ${ }^{29-31}$ In contrast, at the outer leaflet the same membrane curvature is considered to be positive rather than negative (Figure 8). Therefore, the induced negative curvature by aggregates of GM1s could lead to the recruitment of I-BAR domain proteins (Figure 8). To conclude, the outward membrane bending during the initial growth of a membrane nanotube may be generated by GM1s and I-BARs at the outer and inner leaflets of cell membranes, respectively. Further, the attached I-BAR proteins may induce actin self-assembly and in this way promote the elongation of membrane nanotubes (Figure 8). ${ }^{32}$

While the addition of CTB in conjunction with antiCTB is widely used as a marker for lipid ordered domains in cells, ${ }^{14,15}$ the functional effects of formed microdomains of GM1s, CTB, and anti-CTB are not clear. We here suggest that anti-CTB binds more than one CTB molecule, thereby forming membrane microdomains or protein networks, which not only increase the membrane bending rigidity but also the 


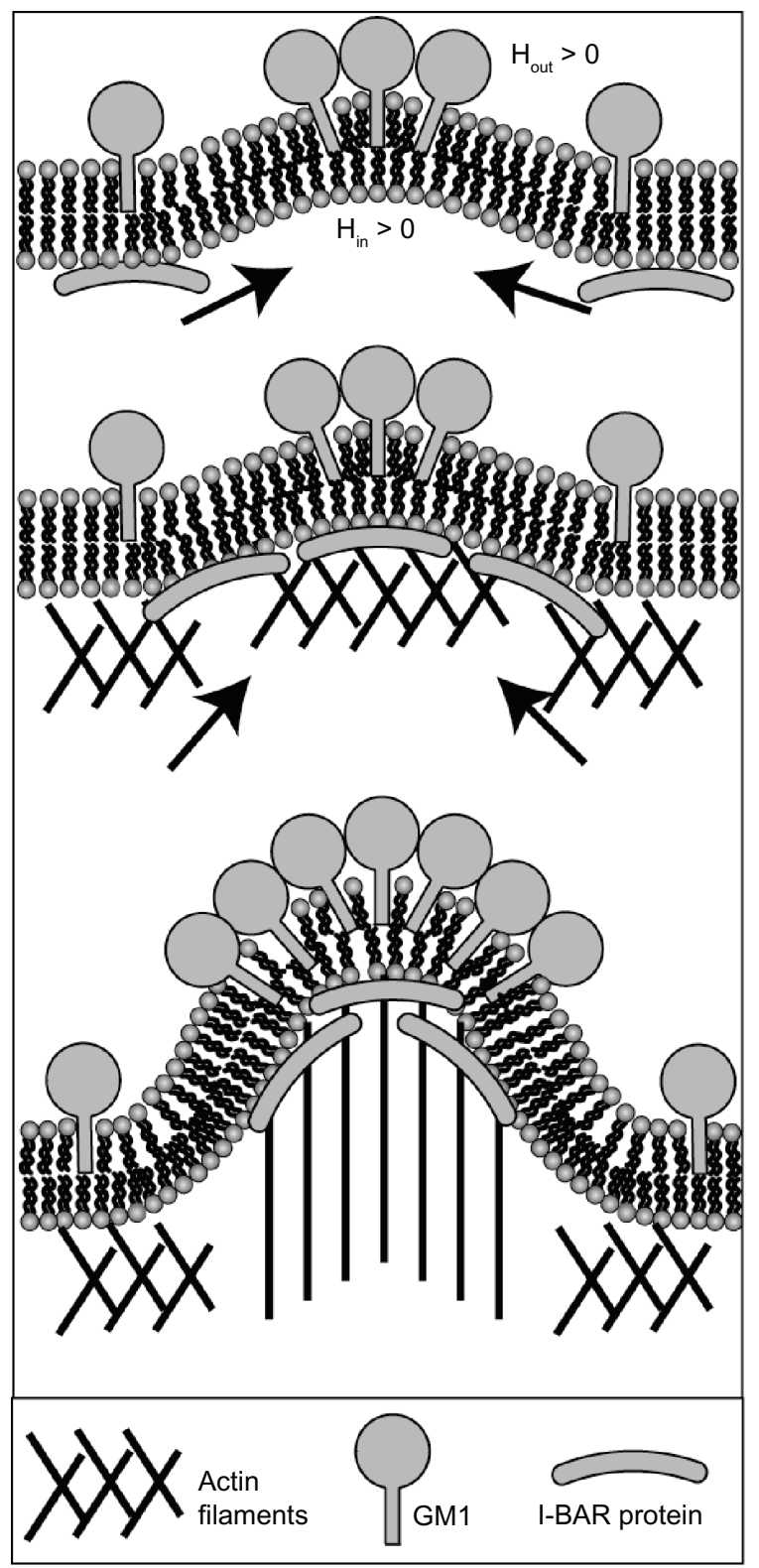

Figure 8 Schematic diagram for the recruitment of I-BAR domain proteins and actin filaments by GMI aggregates. The positive and negative curvatures at the outer and inner leaflets are induced by a GMI aggregate. Consequently, I-BAR domain proteins are attracted to the negative curvature at the inner leaflet of a cell membrane. The I-BAR domain proteins would further bend the membrane, while activating the actin nucleation machinery. The nucleation of actin filaments would drive a membrane protrusive growth, elongating the membrane protrusion.

membrane shear modulus (Figure 7D). ${ }^{18,33}$ As a result, the bound network of CTB molecules at the membrane would prevent the formation of new membrane nanotubes as well as destabilize those already formed.

In the present study, it was also revealed that lowering the temperature of the medium may cause the retraction of ICNs in T24 cells (Figure 2). The effects of temperature in RT4 cells are not as clear, since the corresponding changes in
ICN densities due to temperature change are not significant. The possible explanation for temperature-dependent changes in T24 cells is that a temperature change in the growth medium may alter the cell metabolism mediating the mechanisms responsible for the growth of membrane nanotubes. In addition, the temperature treatment may reduce or increase the fluidity of the membrane to a level that is not optimal for the binding of membrane proteins at the highly curved regions of ICNs. In addition, the application of temperature (eg, cooling to room temperature) might cause the excessive internalization of CTB-GM1 complexes, leading to the depletion of GM1 molecules from the outer leaflet of the cell membrane.

Lipid rafts are suggested to be detergent-resistant microdomains that are enriched with cholesterol and glycosphingolipids (eg, GM1) molecules. Various enzymes and membrane receptors, crucial for intracellular signaling and cell survival, are also found in lipid rafts. Previously, it has been demonstrated that malignant cancer cells have high cholesterol content in their cell membranes, leading to increased densities of lipid rafts. ${ }^{34}$ Furthermore, the depletion of cholesterol levels in malignant cancer cells did cause their eventual cell death, while the other noncancer cells were less sensitive to cholesterol depletion. ${ }^{34}$ Accordingly, malignant cancer cells were shown to be more sensitive to raft binding toxins, suggesting the development of novel therapeutic drugs that target the organization of lipid rafts. ${ }^{34,35}$ The results of the present study indicate that the high fluorescence label at the tip of membrane nanotubes may demonstrate the accumulation of lipid rafts. Thus, it remains to be elucidated whether the cholesterol and GM1 cell contents, which are important constituents of lipid rafts in urothelial cancer cells, are greater than those of their normal counterparts. Finally, due to the high content of lipid rafts in prostate and breast cancer cells, ${ }^{36,37}$ the membrane composition and spatial organization of membrane constituents within lipid rafts might also be altered.

According to X-ray crystallography structures of CT bound to GM1 pentasacharide, only the B subunits bind the GM1 molecules, while the A subunit is important for the toxicity of the CT. ${ }^{14,15}$ Nonetheless, the endocytosis of CTB-GM1 membrane complexes and their further transport towards the endoplasmatic reticulum might cause the reorganization of the actin cytoskeleton, leading to cell damage. ${ }^{15}$ Using actin filament fluorescence markers (phalloidin-FITC), the present study has revealed the integrity of actin filaments, indicating that the CTB-GM1 complexes are probably not 
internalized at room temperature (Figure 3). In contrast, the addition of CTB at physiologic temperature does show reorganization of the actin cytoskeleton, which might be due to enhanced internalization of CTB-GM1 complexes.

The experimental results and theoretical discussion of the present study set the stage for future experiments that could test the biophysical mechanisms responsible for the observed alterations in the frequency of ICNs in RT4 and T24 cancer cell lines. Experiments can be designed to reveal changes in the distribution of membrane constituents and to evaluate the efficacy of lipid raft toxins in normal and cancer cells. In particular, there could be a difference in the numbers of GM1 rafts between RT4 and T24 urothelial cancer cells, explaining the CTB-dependent increases in the densities of membrane nanotubes. To conclude, the relationship between the formation of membrane nanotubes and malignancy of the cell could be important not only for the basic knowledge of cellular function but also the development of therapeutic technology for cancer treatment in the next generation.

\section{Acknowledgments}

This work was supported by ARRS grants J3-9219-0381 and P2-0232-1538.

The authors are grateful to Henry Hägerstrand and Nir Gov for useful discussions.

\section{Disclosure}

The authors disclose no conflicts of interest.

\section{References}

1. Gerdes H, Carvalho R. Intercellular transfer mediated by tunneling nanotubes. Curr Opin Cell Biol. 2008;20:470-475.

2. Gürke S, Barroso J, Gerdes H. The art of cellular communication: tunneling nanotubes bridge the divide. Histochem Cell Biol. 2008;129: 539-550.

3. Sherer N, Mothes W. Cytonemes and tunneling nanotubules in cell-cell communication and viral pathogenesis. Trends Cell Biol. 2008;9:414-420.

4. Lokar M, Perutková S, Kralj-Iglič V, Iglič A, Veranič P. Nanotubes in urothelial cell line T24. In: Advances in Planar Lipid Bilayers and Liposomes: 10th vol. Amsterdam, The Netherlands; Elsevier. 2009:65-94.

5. Hurtig J, Chiu D, Daniel T, Onfelt B. Intercellular nanotubes: insights from imaging studies and beyond. Wiley Interdiscip Rev Nanomed Nanobiotech. 2010;2:260-276.

6. Gerdes H, Bukoreshtliev N, Barroso J. Tunneling nanotubes: a new route for the exchange of components between animal cells. FEBS Lett. 2007;581:2194-2201.

7. Davies D, Sowinski S. Membrane nanotubes: dynamic long-distance connections between animal cells. Nat Rev Mol Cell Biol. 2008;9: 431-436.

8. Veranič P, Lokar M, Schuetz G, et al. Different types of cell-to-cell connections mediated by nanotubular structures. Biophys J. 2008;95: $4416-4425$.
9. Kralj-Iglič V, Heinrich V, Svetina S, Žekš B. Free energy of closed membrane with anisotropic inclusions. Eur Phys J B. 1999; 10:5-8.

10. Iglič A, Hägerstrand $\mathrm{H}$, Veranič $\mathrm{P}$, Plemenitaš A, Kralj-Iglič V. Curvature induced accumulation of anisotropic membrane components and raft formation in cylindrical membrane protrusions. $J$ Theor Biol. 2006;240:368-373.

11. Iglič A, Slivnik T, Kralj-Iglič V. Elastic properties of biological membranes influenced by attached proteins. J Biomech. 2007;40: 2492-2500.

12. Perutková S, Kralj-Iglič V, Frank M, Iglič, A. Mechanical stability of membrane nanotubular protrusions influenced by attachment of flexible rod-like proteins. J Biomech. 2010;43:1612-1617.

13. Kralj-Iglič V, Iglič A, Hägerstrand H, Peterlin P. Stable tubular microexovesicles of the erythrocyte membrane induced by dimeric amphiphiles. Phys Rev E. 2000;61:4230-4234.

14. Holmgren J, Lonnroth I, Svennerholm L. Tissue receptor for cholera exotoxin, postulated structure from studies with GM1 ganglioside and related glycolipids. Infect Immun. 1973;8:208-214.

15. Merritt E, Sarfaty S, van den Akker F, L'Hoir C, Martial J, Hol W. Crystal structure of cholera toxin B-pentamer bound to receptor GM1 pentasaccharide. Protein Sci. 1994;3:166-175.

16. Wernick N, Chinnapen D, Cho J, Lencer W. Cholera toxin: an intracellular journey into the cytosol by way of the endoplasmic reticulum. Toxins. 2010;2:310-325.

17. Alberts A, Johnson A, Lewis J, Raff M, Roberts K, Wlater P. Molecular Biology of the Cell. 5th ed. New York: Gerland Science; 2008:1255.

18. Hägerstrand H, Kralj-Iglič V, Bobrowska-Hägerstrand M, Iglič A. Membrane skeleton detachment in spherical and cylindrical microexovesicles. Bull of Math Biol. 1999;61:1019-1030.

19. Pei B, Chen J. More ordered, convex ganglioside-enriched membrane domains: The effects of GM1 on sphingomyelin bilayers containing a low level of cholesterol. J Biochem. 2003;134:575-581.

20. Veksler A, Gov NS. Phase transitions of the coupled membranecytoskeleton modify cellular shape. Biophys J. 2007;93: 3798-3810.

21. Kabaso D, Shlomovitz R, Auth T, Lew VL, Gov NS. Curling and local shape changes of red blood cell membranes driven by cytoskeletal reorganization. Biophys J. 2010;99:808-816.

22. Kabaso D, Gongadze E, Perutková S, et al. Mechanics and electrostatics of the interactions between osteoblasts and titanium surface. Comput Methods Biomech Biomed Engin. 2011. In press.

23. Cai W, Lubensky TC. Covariant hydrodynamics of fluid membranes. Phys Rev Lett. 1994;73:1186-1189.

24. Helfrich W. Elastic properties of lipid bilayers: theory and possible experiments. Z Naturforsch C. 1973;28:693-703.

25. Romer W, Berland L, Chambon V, et al. Shiga toxin induces tubular membrane invaginations for its uptake into cells. Nature. 2007;450: 670-675.

26. Ewers H, Römer W, Smith AE, et al. GM1structure determines SV40induced membrane invagination and infection. Nat Cell Biol. 2010;12: $11-20$.

27. Sonnino S, Mauri L, Chigorno V, Prinetti A. Gangliosides as components of lipid membrane domains. Glycobiology. 2006;17:1-13.

28. Hägerstrand A, Mrowczynska L, Salzer U, et al. Curvature dependent lateral distribution of raft markers in the human erythrocyte membrane. Mol Membr Biol. 2006;23:277-288.

29. Millard T, Bompard G, Heung M, et al. Structural basis of Filopodia formation induced by the IRSp53/MIM homology domain of human IRSp53. EMBO J. 2005;24:240-250.

30. Mattila PK, Pykalainen A, Saarikangas J, et al. Missing-in-metastasis and IRSp53 deform PI(4,5)P2-rich membranes by an inverse BAR domain-like mechanism. J Cell Biol. 2007;176:953-964.

31. Scita G, Confalonieri S, Lappalainen P, Suetsugu S. IRSp53: crossing the road of membrane and actin dynamics in the formation of membrane protrusions. Trends Cell Biol. 2008;18:52-60. 
32. Zhao H, Pykäläinen A, Lappalainen P. I-BAR domain proteins: linking actin and plasma membrane dynamics. Curr Opin Cell Biol. 2010;23:1-8.

33. Schara K, Jansa V, Šuštar V, et al. Mechanisms for the formation of membranous nanostructures in cell-to-cell communication. Cell Mol Biol Lett. 2009; 14:636-656.

34. Li Y, Park M, Ye S, Kim C, Kim Y. Elevated levels of cholesterol-rich lipid rafts in cancer cells are correlated with apoptosis sensitivity induced by cholesterol-depleting agents. Am J Pathol. 2006;168:1107-1118.
35. Johannes L, Decaudin D. Protein toxins: intracellular trafficking for targeted therapy. Gene Therapy. 2005:12:1360-1368.

36. Zhuang L, Lin J, Lu M, Solomon K, Freeman M. Cholesterol-rich lipid rafts mediate Akt-regulated survival in prostate cancer cells. Cancer Res. 2002;62:2227-2231.

37. Sarnataro D, Grimaldi C, Pisanti S, et al. Plasma membrane and lysosomal localization of CB1 cannabinoid receptor are dependent on lipid rafts and regulated by anandamide in human breast cancer cells. FEBS Lett. 2005;579:6343-6349. 


\section{Supplementary material}

\section{The model}

To test the hypothesis that the initial formation of a membrane nanotube is driven by the aggregation of CTB-GM1 complexes, we constructed a model to study the effects of strong binding of CTB to GM1 molecules. The model is an extension of previous theoretical work,,$^{20,22}$ and the present aim is to test whether the less positive spontaneous curvature of CTB-GM1 complexes as well as the strong binding of CTB are sufficient to drive an instability causing the growth and coalescence of membrane protrusions.

Our model is a coarse-grained model, whereby we do not describe the detail of the molecular-scale level. The minimal length-scale along the membrane that is relevant to this model is of the order of $100 \mathrm{~nm}$. The model is written as a set of equations of motion for the continuum fields that describe the membrane shape and density of CTB-GM1 complexes, including the actual forces acting on the membrane, and the details of the membrane elasticity. For the sake of simplicity, the modeled membrane contour of RT4 or T24 cells is considered to be nearly flat. The bending energy part of the free energy of the model is based on the Helfrich energy form. ${ }^{24}$ The free energy expression includes also the CTB-GM1 binding, configurational entropy, and direct-interaction energy:

$$
\begin{aligned}
F= & \int\left(\frac{1}{2} \kappa(H-\bar{H} n)^{2}+(\sigma-\alpha n)+\gamma h^{2}\right. \\
& \left.+k_{B} T n_{s} n(\ln (n)-1)-J n^{2}+\frac{J}{n_{s}}(\nabla n)^{2}\right) d s,
\end{aligned}
$$

where $k$ is the membrane bending rigidity, $n$ is CTB-GM1 density, $H$ is the local mean membrane curvature, $\bar{H}$ is the parameter describing the intrinsic curvature of the CTB-GM1s, $n_{s}$ is the saturation density of CTB-GM1s on the membrane, $\sigma$ is the membrane surface tension, $\alpha$ is a proportionality constant describing the effective adhesion interaction between CTB and GM1s, $h=h(x)$ describes the shape of the contour, $\gamma$ is a restoring force of cytoskeleton, $J$ is the direct nearest-neighbor interaction energy between CTB and GM1s, and $d s=d_{m} \cdot d l$ is an element of membrane area, where $d_{m}$ is the dimension of membrane perpendicular to the contour and $d l$ is a line element along the contour. Note that $k$ equals 4 times the $k$ used in Helfrich. ${ }^{24}$

The first term gives the bending energy due to the mismatch between the membrane curvature and the spontaneous curvature of a CTB-GM1 complex. The term $-\alpha n$ describes the negative adhesion energy between CTB and GM1s. The third term describes an external trapping of the membrane, which can be represented as the force of the cytoskeleton. The fourth term gives the entropic contribution due to the lateral thermal motion of the CTB-GM1 in the membrane in the limit of small $n$. The fifth term describes the nearest-neighbor attractive interactions between CTB and GM1s, and the sixth term prevents the sharp jumps in $n$.

\section{Derivation of the curvature force on the membrane}

We derive the equations of motions of the membrane contour using the derivation of the free energy [Eq. (1)] with respect to the membrane coordinate and CTB-GM1 concentration. ${ }^{21}$ To take into account the drag due to viscous forces, we assume for simplicity only local friction forces, ${ }^{20,21}$ with coefficient $\xi$.

The equation of motion of the membrane is

$$
\xi \frac{\partial \vec{r}}{\partial t} \cdot \vec{n}=-\frac{\delta F(s, t)}{\delta n}
$$

where $\xi$ is the coefficient of the local friction force due to viscous drag of the fluids surrounding the membrane, $\vec{r}$ is the radial vector in the $(x, y)$ coordinate system, $t$ is time, $\vec{n}$ is the normal direction, and $\delta F(s, t) / \delta n$ is the derivation of Eq. (1) with respect to the $x$ and $y$ directions. Here we consider only the changes along the $y$ direction. The derivation of the free energy is projected to give the forces normal to the membrane contour. ${ }^{21}$ The following is the list of parameter values incorporated in our model: $\xi=125 \mathrm{~s}^{-1} \mathrm{gr}, \mathrm{D}=0.002 \mu \mathrm{m}^{2} \mathrm{~s}^{-1}, \Lambda=\mathrm{D} / \mathrm{k}_{\mathrm{B}} \mathrm{T}, \alpha=0.013 \mathrm{gr} \mathrm{s}^{-2}$, $\gamma=0.0004 \mathrm{grs}^{-2}, \mathrm{n}_{\mathrm{s}}=10 \mu \mathrm{m}^{-2}, k=100 \mathrm{k}_{\mathrm{B}} \mathrm{T}, \bar{H}=5 \mu \mathrm{m}^{-1}$, $J=0.00035 \mathrm{grs}^{-2}$, and $\sigma=0.001 \mathrm{grs}^{-2}$.

We next derive the forces at the membrane, by treating it as a "one-dimensional membrane", ie, a thin strip of width $w$, with a bending modulus and tension coefficient. The free energy of this membrane is given in Eq. (1), and is used to derive the local restoring forces by the usual derivation method. Since the overall contour length is not constant in our system, the derivation of the coordinates has to be taken with respect to their absolute index $u$ along the contour, which is constant. In these terms the curvature $H$ appearing in the Helfrich part of the free energy ${ }^{24}$ is written as (standard differential geometry)

$$
H=\frac{\ddot{x} \ddot{y}-\dddot{x} \ddot{y}}{\sqrt{\dot{x}^{2}+\dot{y}^{2}}},
$$


where the $\cdot$ symbol denotes differentiation with respect to the index of the point along the contour, and the free energy is

$$
F=w \int\left(\frac{1}{2} \kappa H^{2}+\sigma\right) \sqrt{\dot{x}^{2}+\dot{y}^{2}} d u,
$$

where $d s / d u=\dot{s}=\sqrt{\dot{x}^{2}+\dot{y}^{2}}$. The derivation of this free energy gives the forces, for example in the $x$-direction

$$
F_{x}=-\frac{\delta F}{\delta x}=\frac{d}{d u} \frac{\delta F}{\delta \dot{x}}-\frac{d^{2}}{d u^{2}} \frac{\partial F}{\partial \ddot{x}} .
$$

The resulting equations of motion from this derivation gives very long expressions, which are not amenable to easy analysis, although they can be used for the numerical simulations. In order to arrive at simpler expressions we will develop the terms in Eq. (5), and simplify at the end by assuming that the arc-length separation between the nodes along the contour are all the same. This is maintained as the simulation progresses by using the spline routine to rediscretize evenly the contour as its length evolves.

The first term on the r.h.s. of Eq. (5) is

$$
\frac{\delta F}{\delta \dot{x}}=H^{2} \frac{\delta x}{\delta s}+2 H \frac{\partial H}{\partial \dot{x}} \dot{s}
$$

where $\frac{\delta \dot{x}}{\delta \dot{s}}=\dot{x} / \sqrt{\dot{x}^{2}+\dot{y}^{2}}=\frac{\partial x}{\partial s}$.

$$
\begin{aligned}
\frac{d}{d u} \frac{\delta F}{\delta \dot{x}}= & 2 \dot{s} H H^{\prime} x^{\prime}+\dot{s} H^{2} x^{\prime \prime}+2 \dot{s}^{2} H^{\prime} \frac{\partial H}{\partial \dot{x}} \\
& +2 H \frac{\partial H}{\partial \dot{x}} \ddot{s}+2 H \dot{s}^{2} \frac{\partial H^{\prime}}{\partial \dot{x}}
\end{aligned}
$$

where $\partial H / \partial \dot{x}=\ddot{y} \dot{s}^{3}-3 x^{\prime} H / \dot{s}$. We therefore need to find an expression for $\ddot{y}$ (and $\ddot{x}$ ), by using the definition of $H=\left(x^{\prime} \ddot{y}-y^{\prime} \ddot{x}\right) / \dot{s}^{2}$ and: $\ddot{s}=x^{\prime} \ddot{x}+y^{\prime} \ddot{y}$. The final expressions that we get are: $\ddot{y}=\ddot{s} y^{\prime}+\dot{s}^{2} x^{\prime} H$, and $\ddot{x}=\ddot{s} x^{\prime}-\dot{s}^{2} y^{\prime} H$. We will now assume that $\dot{s}$ is independent of $u$, so that $\ddot{s}=\dddot{s}=0$. The last term in Eq. (7) becomes

$$
\frac{\partial H^{\prime}}{\partial \dot{x}}=-\frac{2}{\dot{s}} \frac{\partial}{\partial s}\left(x^{\prime} H\right)
$$

The second term on the right hand side of Eq. (5) is

$$
\frac{\partial F}{\partial \ddot{x}}=\frac{-2 H y^{\prime}}{\dot{s}}
$$

and we get

$$
\frac{d^{2}}{d u^{2}} \frac{\partial F}{\partial \ddot{x}}=-2 \dot{s} \frac{\partial^{2}}{\partial s^{2}}\left(H y^{\prime}\right)
$$

We now write $H$ in terms of $s$ as: $H=\vec{H} \cdot \hat{n}=-x^{\prime \prime} y^{\prime}+y^{\prime \prime} x^{\prime}$, where: $\vec{H}=x^{\prime \prime} \hat{x}+y^{\prime \prime} \hat{y}$, and $\hat{n}=-y^{\prime} \hat{x}+x^{\prime} \hat{y}$ so that

$$
\begin{gathered}
H^{\prime}=-y^{\prime} x^{\prime \prime \prime}+x^{\prime} y^{\prime \prime \prime} \\
H^{\prime \prime}=-y^{\prime \prime} x^{\prime \prime \prime}+x^{\prime \prime} y^{\prime \prime \prime}-y^{\prime} x^{(4)}+x^{\prime} y^{(4)}
\end{gathered}
$$

There is another force contribution from the membrane tension, giving a term of the form: $F_{x} \propto \dot{s} x^{\prime \prime}$, so in the normal direction we get: $F_{n}=\dot{s} \sigma\left(-y^{\prime} x^{\prime \prime}+x^{\prime} y^{\prime \prime}\right)=\dot{s} \sigma H$, where we used the identity: $x^{\prime 2}+y^{\prime 2}=1$ and therefore: $2\left(x^{\prime} x^{\prime \prime}+y^{\prime} y^{\prime \prime}\right)=\partial\left(x^{\prime 2}+y^{\prime 2}\right) / \partial s=0$.

Putting everything together, the normal force acting on the membrane due to curvature and tension is

$$
\begin{gathered}
F=\vec{F} \cdot \hat{n}=-y^{\prime} F_{x}+x^{\prime} F_{y} \\
=\dot{s} \frac{1}{2} \kappa\left(-2 \nabla^{2} H-2 H\left(y^{\prime} y^{\prime \prime \prime}+x^{\prime} x^{\prime \prime \prime}\right)\right. \\
\left.-3(\vec{H} \cdot n)^{3}\right)+\dot{s} \sigma H \\
=\dot{s} \kappa\left(-\nabla^{2} H-\frac{1}{2}(\vec{H} \cdot \hat{n})^{3}\right)+\dot{s} \sigma H \\
\dot{s} \frac{1}{2} \kappa\left(2\left(y^{\prime} x^{(4)}-x^{\prime} y^{(4)}\right)-3(\vec{H} \cdot n)^{3}\right)+\dot{s} \sigma H
\end{gathered}
$$

where we used the identity: $x^{\prime 2}+y^{\prime 2}=1$, and therefore:

$2\left(x^{\prime} x^{\prime \prime}+y^{\prime} y^{\prime \prime}\right)=\partial\left(x^{\prime 2}+y^{\prime 2}\right) / \partial \mathrm{s}=0$, and $-\left(x^{\prime \prime 2}+y^{\prime \prime 2}\right)=-H^{2}$

We need forces per unit length, whereas we calculated above the forces per unit $u$, so we divide by $\dot{s}$ and finally get

$$
\kappa\left(\left(y^{\prime} x^{(4)}-x^{\prime} y^{(4)}\right)-\frac{3}{2} H^{3}\right)+\sigma H .
$$

CTB-GM1s with spontaneous curvature

When there are CTB-GM1s with spontaneous curvature, the free energy.

(Eq. (4)) changes to

$$
F=w \int^{1}\left(\kappa(H-\bar{H} n)^{2}+(\sigma-\alpha n)\right) \sqrt{\dot{x}^{2}+\dot{y}^{2}} d u
$$

where $n$ the density of CTB-GM1s along the contour, which may not be uniform. Expanding the quadratic term we get: $H^{2}-2 H \bar{H} n+(\bar{H} n)^{2}$. The derivation of the first term was done above (all the variations are of the integrand times the $\dot{s}$ factor).

The new contributions to the forces acting on the membrane are (normal force per unit length)

$$
\begin{aligned}
F_{\text {spon }, n} & =\vec{F}_{\text {spon }} \cdot \hat{n}=-y^{\prime} F_{\text {spon }, x}+x^{\prime} F_{\text {spon }, y} \\
& =\frac{K}{2}\left((\bar{H} n)^{2} H-2\left(\bar{H} n^{\prime \prime}\right)-n \bar{H} H^{2}\right)
\end{aligned}
$$




$$
F_{\text {tension }, n}=\vec{F}_{\text {spon }} \cdot \hat{n}=-\alpha n H .
$$

Fluxes and diffusion of CTB-GM1s

The conservation equation for the CTB-GM1s along the contour becomes

$$
\frac{1}{\dot{s}} \frac{\partial \dot{s} n}{\partial t}=\frac{D}{\dot{s}} \nabla_{s}^{2}(\dot{s} n)+\frac{1}{\dot{s}} \frac{\Lambda}{n_{s}} \nabla_{s}(\dot{s} n) \nabla_{s}\left(\frac{1}{\dot{s}} \frac{\delta F}{\delta n}\right),
$$

where the number of CTB-GM1s in each unit contour length is $N=\dot{s} n n_{s}$ ( $n_{s}$ is the saturation concentration of the CTBGM1s), $F$ is the energy functional of Eq. (1), and the derivative along the contour is $\nabla_{s}=\nabla_{u} / \dot{s}$. We therefore get

$$
\frac{\partial n}{\partial t}+\frac{n}{\dot{s}} \frac{\partial \dot{s}}{\partial t}=\frac{D}{\dot{s}} \nabla_{s}^{2}(\dot{s} n)+\frac{D}{k_{B} T n_{s} \dot{s}^{2}} \nabla_{u}\left(n \nabla_{u} \frac{\delta F}{\delta n}\right)
$$

where $e$ is the energy per unit length, ie, the integrand in Eq. (15) with respect to $d s$.

If the number of CTB-GM1s is conserved, even though we allow the membrane overall length to change, then Eq. (19) is correct. If however there is a reservoir of membrane that allows it to change in length, then this membrane can include lipids and CTB-GM1s, so that the total number of CTB-GM1s is not conserved when the membrane length changes. In this case the change in the density due to length changes is removed, as it is assumed to be balanced by the currents into/out of the reservoir. Eq. (19) is then modified by removing the second term on the left hand side.

In our calculations, a nonlinear tension was employed, and as a result, the length of each membrane segment changed very little, so the second term on the left hand side of Eq. (19) was neglected.

\section{Numerical realization of the model Discretization of the model}

Since the flat shape model represents a segment of the whole cell, we used periodic boundary conditions. Thus, the number of grid points $N$ equals the number of discretizations. In our model, the density $n$ of element $i$ is given by:

$$
n_{i}=\frac{N_{i}}{\Delta s_{i}}
$$

\section{The boundary conditions}

We employed periodic boundary conditions. The first and second derivatives of the function along the $x$ direction were calculated using the following explicit Euler method:

$$
\frac{\partial x}{\partial s}=\frac{x_{n+1}-x_{n-1}}{2 \Delta s_{i}}
$$

$$
\frac{\partial^{2} x}{\partial s^{2}}=\frac{x_{n-1}-2 x_{n}+x_{n+1}}{\Delta s_{i}^{2}}
$$

where the subscripts $n, n+1, n-1$ represent the current, next, and previous nodes, respectively. The derivatives of the function along the $y$ direction were calculated in a similar manner. For the calculations of derivatives of the first point, the last point was added before it. While for the calculation of derivatives of the last point, the first point was added after it.

\section{The derivation of the free energy equation}

The derivation of the free energy is projected to give the forces normal to the membrane contour. ${ }^{21}$ We now list the forces derived from the derivation of the free energy [Eq. (1) ${ }^{21}$

$$
\begin{gathered}
F_{c}=\kappa\left(-\nabla^{2} H+\bar{H} \nabla^{2} n+\frac{1}{2} n^{2} \bar{H}^{2} H-\frac{1}{2} H^{3}\right) \\
F_{t}=(\sigma-\alpha n) H \\
F_{s}=-2 \gamma y \\
F_{e}=k_{B} T n_{s}(n \ln (n)-1) H \\
F_{J}=J\left(-n^{2}+\frac{1}{n_{s}}(\nabla n)^{2}\right) H
\end{gathered}
$$

where $F_{c}$ is the force due to the curvature energy mismatch between the membrane curvature and the spontaneous curvature of the CTB-GM1s, $F_{t}$ is the membrane tension force, $F_{s}$ is the spring restoring force, and $F_{J}$ is the force due to the nearest-neighbor interaction of the CTB-GM1s. $F_{e}$ arises from the entropy of the CTB-GM1s in the membrane, which acts to expand the length of the contour.

We now calculate the dynamics of the CTB-GM1 density, using the following conservation equation

$$
\frac{\partial n}{\partial t}=-\nabla \cdot \vec{J}=\frac{\Lambda}{n_{s}} \nabla_{s}\left(n \nabla_{s} \frac{\delta F}{\delta n}\right)-\frac{n}{\sqrt{g}} \frac{\partial \sqrt{g}}{\partial t},
$$

where $\Lambda$ is the mobility of CTB-GM1s and $\vec{J}$ is the total current of CTB-GM1 on the membrane, which includes the following terms

$$
\begin{gathered}
J_{\text {att }}=\frac{\kappa \Lambda \bar{H}}{n_{s}} n \nabla H \\
J_{\text {disp }}=-\frac{\kappa \Lambda \bar{H}^{2}}{n_{s}} n \nabla n
\end{gathered}
$$




$$
\begin{gathered}
J_{\text {agg }}=\frac{J \Lambda}{n_{s}} n \nabla n+\frac{J \Lambda}{n_{s}{ }^{2}} n \nabla^{3} n \\
J_{d i f f}=-D \nabla n
\end{gathered}
$$

where $J_{\text {att }}$ is the attraction flux resulting from the interaction between the CTB-GM1s through the membrane curvature, $J_{\text {disp }}$ is the dispersion flux due to the membrane resistance to CTB-GM1 aggregation due to their membrane bending effects, $J_{a g g}$ is the flux due to the direct CTB-GM1 aggregation interactions, and $J_{\text {diff }}$ is the usual thermal diffusion flux, which depends on the diffusion coefficient, $D=\Lambda \mathrm{k}_{\mathrm{B}} T$. The last term in Eq. (27) arises from the covariant derivative of the density with time on a contour whose length evolves with time. ${ }^{23}$ In this term $\sqrt{g}$ is the matrix tensor, which in our onedimensional contour is simply the line element $d l$. This term ensures that the total number of CTB-GM1s is conserved as the contour length changes.

\section{Publish your work in this journal}

The International Journal of Nanomedicine is an international, peerreviewed journal focusing on the application of nanotechnology in diagnostics, therapeutics, and drug delivery systems throughou the biomedical field. This journal is indexed on PubMed Central, MedLine, CAS, SciSearch ${ }^{\circledR}$, Current Contents ${ }^{\circledR} /$ Clinical Medicine,
Journal Citation Reports/Science Edition, EMBase, Scopus and the Elsevier Bibliographic databases. The manuscript management system is completely online and includes a very quick and fair peer-review system, which is all easy to use. Visit http://www.dovepress.com/ testimonials.php to read real quotes from published authors. 